\title{
Visualization of Geodynamo Simulations
}

\author{
Amit Chourasia \\ UC San Diego \\ amit@sdsc.edu
}

\section{Sam Greenwood University of Leeds ee12sg@leeds.ac.uk}

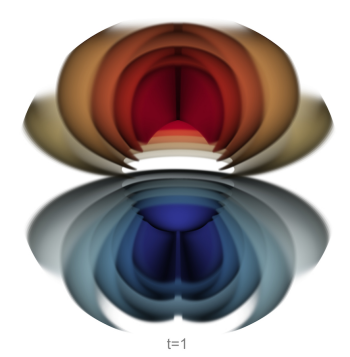

\author{
Maggie Avery \\ UC San Diego \\ msavery@ucsd.edu
}

\author{
Ashley Willis \\ University of Sheffield \\ a.p.willis@sheffield.ac.uk
}

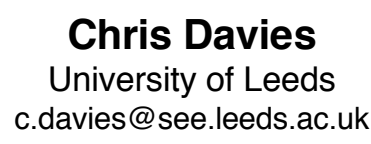
Catherine Constable UC San Diego
cconstable@ucsd.edu
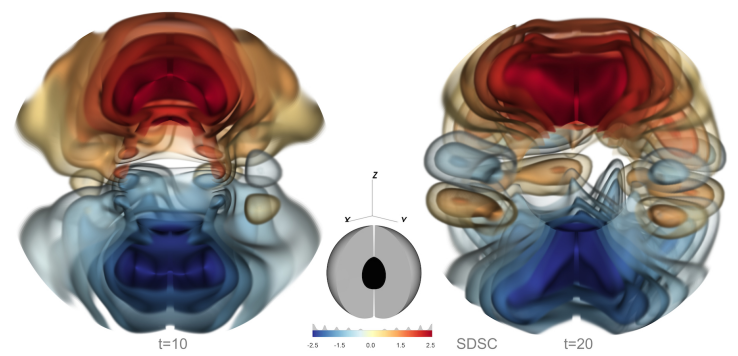

David Gubbins

University of Leeds gubbins@earth.leeds.ac.uk

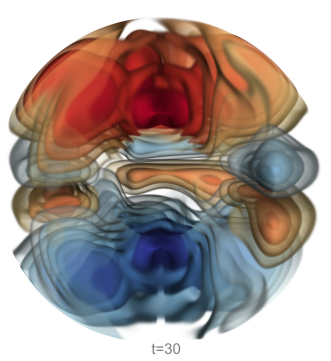

Figure 1. The image shows evolution of radial velocity from initial to steady state from left to right. The small inset image at the center indicates the section of the sphere being displayed in larger images.

\begin{abstract}
Earth's magnetic field undergoes striking variations in direction and strength on a vast spectrum of time scales. Complex motions of the liquid iron core maintain the magnetic field, but the inner workings of this so-called dynamo process are obscured from direct observation and pose significant challenges for theoretical and computational modeling. This study aims to simulate the dynamo and determine which parameters can be associated with common features that are observed in the incomplete geological record of the magnetic field. The simulation produces an output comprised of magnetic field, the fluid flow, and temperature in the Earth's core. Visualization results based on the output data are compared with observations projected down to the core surface, and to link those features with model behavior deep within the core. The visualization aspect of this study is enabling the scientists to identify interesting physical phenomena in the models that may be related to observation, including fluid up and down welling throughout the liquid core welling throughout the liquid core.
\end{abstract}

Permission to make digital or hard copies of part or all of this work for personal or classroom use is granted without fee provided that copies are not made or distributed for profit or commercial advantage and that copies bear this notice and the full citation on the first page. Copyrights for third-party components of this work must be honored. For all other uses, contact the Owner/Author.

XSEDE16, July 17 - 21, 2016,

Copyright is held by the owner/author(s).

XSEDE16, July 17-21, 2016,

ACM 978-1-4503-4755-6/16/07.

http://dx.doi.org/10.1145/2949550.2952772

\section{CCS Concepts}

Human-centered computing Visualization

$\rightarrow$ Visualization techniques

$\rightarrow$ Visualization application domains

$\rightarrow$ Visualization systems and tools

\section{Keywords}

Geodynamo; HPC; Visualization;

\section{INTRODUCTION}

Earth's magnetic field is produced by a process called the geodynamo, via convective motions in the liquid-iron outer-core, which lies some $2800 \mathrm{~km}$ below the planet's surface. The field has persisted for most of Earth's history and varies in both direction and intensity on a broad range of time scales. On geological time scales sediments and newly forming igneous rocks record the field at Earth's surface and suitable samples provide a window into the dynamics and evolution of the liquid core. Limited reconstructions of the past geomagnetic field can be downward continued from the surface through the electrically insulting mantle to the core mantle boundary, but not inside the highly conductive core. Numerical simulations of the dynamo process can complement the observations. Simulating the geodynamo [1, 2] we can learn about the dynamical processes that generate the field behavior observed at the surface, which may eventually allow forecasting of outer core conditions.

\section{SIMULATION}

The Earth's outer core can be modeled as a rapidly rotating spherical shell filled with an electrically conducting fluid. The shell is usually cooled from above or heated from within, which gives rise to thermal buoyancy forces that drive convection. Compositional buoyancy can also be included to mimic the release of light elements into the liquid on freezing and growth of the solid inner core. Fluid motions induce magnetic fields, which in turn act to oppose the motion via the Lorentz force. Magnetic 
energy is destroyed due to electrical resistance. Under the right conditions induction overcomes dissipation and a self-sustaining dynamo is obtained. Numerical simulations solve the NavierStokes equation with Coriolis and Lorentz force terms, together with the magnetic induction and heat diffusion equations. The flow is assumed incompressible and the field is solenoidal, which provide two divergence-free conditions that close the dynamo equations.

The "Leeds Dynamo Code" [3] solves the dynamo equations in spherical coordinates by representing the fluid velocity and magnetic field in poloidal and toroidal scalars [4]. This representation automatically satisfies the divergence-free conditions. For each scalar the meridional and azimuthal variations are represented in spherical harmonics, and radial variations by variable order, variable mesh, finite differences. Nonlinear terms are evaluated by the transform method: fields are transformed from spectral to real space, nonlinear terms are evaluated at points on a spherical grid, and the results are transformed back. Time stepping is by a predictor-corrector method. The visualizations described here are from a solution that is a community benchmark for testing different computational methods. The solution drifts uniformly in longitude and is well suited for testing new visualization techniques..

\section{VISUALIZATION TECHNIQUES}

The simulation creates a compact output in spectral space that needs to be translated into spatial form, which is subsequently saved in NETCDF format [5] on a known rectilinear grid for visualization with existing tools. The visualizations for this study were performed using VisIt [6]. The data are transformed to a spherical grid using a standard transform operator in VisIt, but it leaves two issues first, the spherical shell does not wrap around along azimuthal direction, second there are no data along the poles. The first issue is resolved by using another standard index operator which allows wrapping of data along desired axis, the second issue of no data along the pole is left as is, due to lack of such operator in VisIt.

Several visualization techniques were utilized for investigating the data. These include color maps of slices and shells at different radius, isosurfaces [7] at desired levels, streamlines [8] and volume renderings [9] for several variables. The visualizations were performed on several simulations on the Stampede cluster at Texas Advanced Computing Center and shared with the scientists using SeedMe.org infrastructure [10]. Representative visualizations are described below.

\subsection{Color mapping with deformation}

A vector field, such as velocity is investigated by comparing different vector components. For example Figure 2, column 1 shows a color map of radial velocity shell, in column two the color mapped radial velocity is deformed by meridional (phi) component of velocity and in column three the color mapped radial velocity if deformed by azimuthal (theta) component of velocity, and the rows indicated different time steps. This composite image allows the scientists to compare and contrast the strength of different components of vector field in relation to each other.

\subsection{Volumetric rendering}

The scalar fields are explored with volume rendering with several transfer functions, which help scientist focus on region of interest. For example Figure $3 \& 4$, middle row shows volumetric renderings of radial magnetic, radial velocity and temperature from left to right.
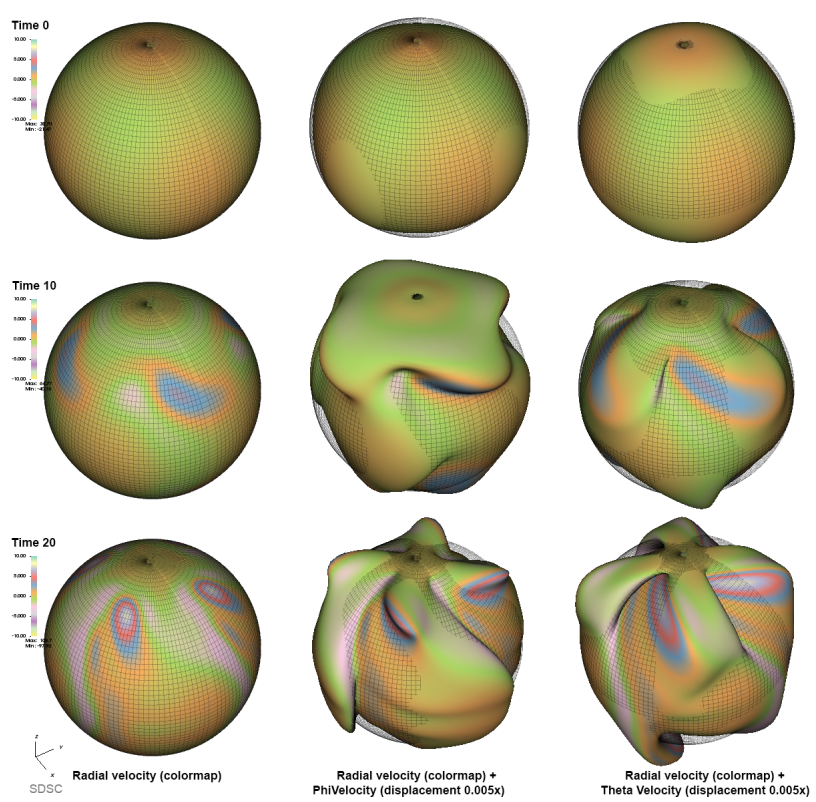

Figure 2. Working downward, Column 1 shows the radial velocity on a spherical shell, while Columns 2 and 3 displays radial velocity with color, with the shell displaced by $P$ Phi and Theta components of velocity fields respectively. The grids provide reference of the original location of the spherical shell.
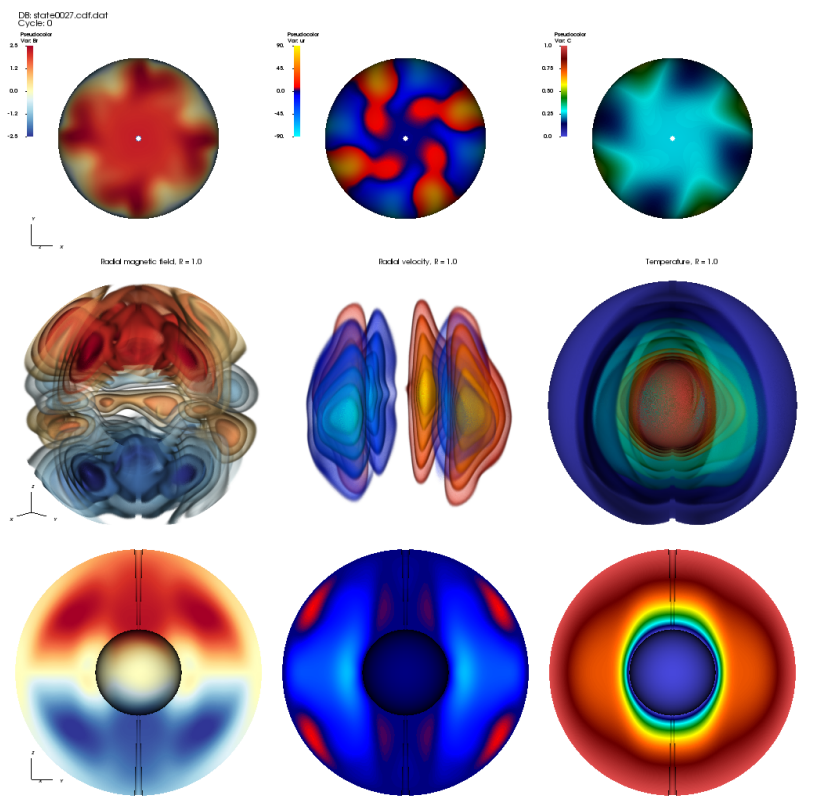

Figure 3. Working downward, Column 1 shows the radial magnetic field on a spherical shell, while Columns 2 and 3 displays radial velocity with color, and Column 3 is temperature. Row 1 looks down at north pole, row 2 volume rendering with wedge cut to outer core, row 3 is meridional slice. 


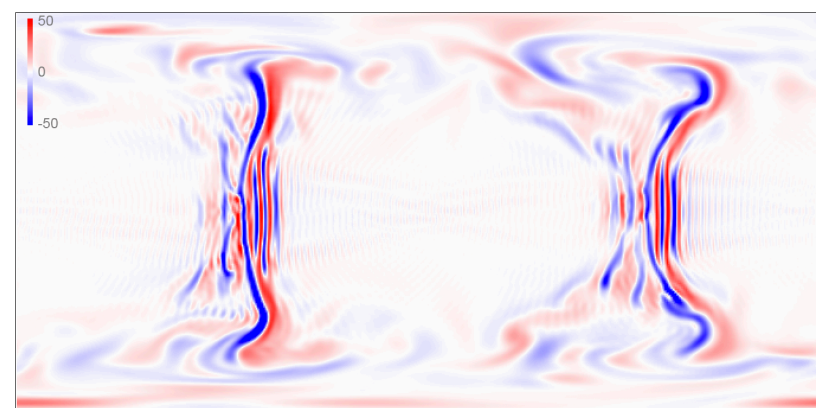

Figure 4. Radial velocity field just below the surface showing a ghost periodic array across convection cells, the cause is being investigated further [2].

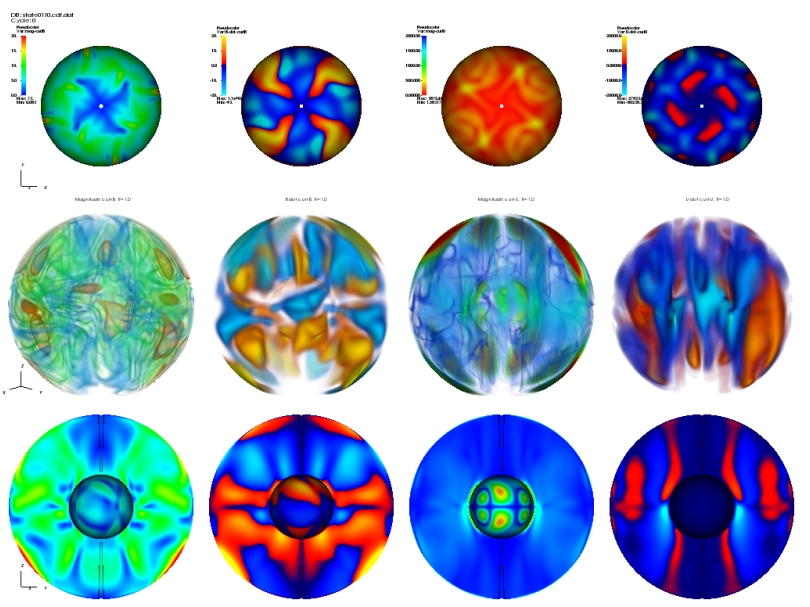

Figure 5. Column $1 \& 2$ show curl magnitude and helicity of magnetic field respectively, and column 3, 4 show curl magnitude and helicity for velocity field. Top row shows shell at radius 1.0, middle row shows volume rendering and bottom row shows meridional section.

\section{RESULTS AND DISCUSSION}

The visualizations have allowed the scientists to re-investigate their existing results in a new and easier manner, exposing the interplay of fluid flow and magnetic field variations. The VisIt software package allows quick generation of high-quality animations of simulated data. In more complex simulations we will compare maps of the simulated and observed radial magnetic field at the outer boundary, looking for simulations with features similar to those seen in the Earth's magnetic field. We will also investigate the fluid velocity, temperature, and magnetic field in the outer core to diagnose the flow configurations associated with the field structures at the boundary, and examine the interplay between diffusion and advection.

\section{ACKNOWLEDGMENTS}

We would like to thank Jean Favre and David Semeraro. This work was supported by NSF award TG-EAR130004 (Tuning Geodynamo Simulations to Paleomagnetic Observations), the Extreme Science and Engineering Discovery Environment (XSEDE) and the XSEDE Extended Collaborative Support
Service (ECSS) program, which is supported by NSF award number ACI-1053575. SeedMe project that is supported by NSF ACI-1235505 and ACI-1443083 awards.

\section{REFERENCES}

[1] Christensen, U.R, J. Aubert, P. Cardin, E. Dormy, S. Gibbons, G.A. Glatzmaier, E. Grote, Y. Honkura, C. Jones, M. Kono, M. Matsushima, A. Sakuraba, F. Takahashi, A. Tilgner, J. Wicht, K. Zhang. 2001. "A numerical dynamo benchmark." Physics of the Earth and Planetary Interiors 128.1 (2001): 25-34.

[2] Sreenivasan, B. and D. Gubbins. 2008. "Dynamos with weakly convecting outer layers: implications for core-mantle boundary interaction." Geophysical and Astrophysical Fluid Dynamics 102.4 (2008): 395-407.

[3] Willis, A.P. , B, Sreenivasan, D.Gubbins (2007) Thermal core-mantle interaction: Exploring regimes for 'locked' dynamo action., Phys. Earth Planet Inter. 165, 83-92, doi:10.1016/j.pepi.2007.08.002.

[4] Backus, G., R. Parker, C. Constable (1996) Foundations of Geomagnetism. Cambridge University Press,

[5] Rew, R. K., G. P. Davis, S. Emmerson, and H. Davies. 1997. NetCDF User's Guide for C, An Interface for Data Access, Version 3, April 1997

[6] Childs, H., E. Brugger, B. Whitlock, J. Meredith, S. Ahern, K. Bonnell, M. Miller, G. H. Weber, C. Harrison, D. Pugmire, T. Fogal, C. Garth, A. Sanderson, E. W. Bethel, M. Durant, D. Camp, J. M. Favre, O. Rübel, Paul Navrátil. 2012. Book chapter: "VisIt: An End-User Tool For Visualizing and Analyzing Very Large Data," in High Performance Visualization: Enabling Extreme-Scale Scientific Insight, edited by E. Wes Bethel, H. Childs, C. Hansen, Chapman and Hall/CRC, October, 2012

[7] Lorensen, W.E., and Harvey E. Cline. 1987. Marching cubes: A high resolution 3D surface construction algorithm. SIGGRAPH Comput. Graph. 21, 4 (August 1987), 163-169. http://doi.acm.org/10.1145/37402.37422

[8] Pugmire, D., H. Childs, C. Garth, S. Ahern, and G. Weber. 2009. Scalable Computation of Streamlines on Very Large Datasets. In Proceedings of Supercomputing, 2009

[9] Levoy. M. 1988. Display of Surfaces from Volume Data. IEEE Comput. Graph. Appl. 8, 3 (May 1988), 29-37. DOI=10.1109/38.511 http://dx.doi.org/10.1109/38.511

[10] Chourasia, A., M. Wong-Barnum, M. Norman. 2013. SeedMe Preview: Your Results from Disk to Device In Proceedings of the Conference on Extreme Science and Engineering Discovery Environment: Gateway to Discovery (XSEDE '13). ACM, New York, NY, USA, , Article 35 , 4 pages. DOI $=10.1145 / 2484762.2484764$

[11] David Thompson, Jeff Braun, and Ray Ford, OpenDX: Paths to Visualization, Vis Inc., 2001.

[12] Chourasia A., 2015. Visualization of a Geodynamo simulation. Retrieved Jun 13, 2016 from https://www.seedme.org/node/40472 